\title{
Multimodal therapy for locally advanced prostate cancer: the roles of radiotherapy, androgen deprivation therapy, and their combination
}

\author{
Sung Uk Lee, MD, Kwan Ho Cho, MD \\ The Proton Therapy Center, Research Institute and Hospital, National Cancer Center, Goyang, Korea
}

Locally advanced prostate cancer (LAPC) is defined as histologically proven T3-4 prostatic adenocarcinoma. In this review, we define the individual roles of radiotherapy (RT), short-term (ST-) and long-term (LT-) androgen deprivation therapy (ADT), and their combination in multimodal therapy for LAPC. Despite limitations in comparing the clinical outcomes among published papers, in the present study, a trend of 10-year clinical outcomes was roughly estimated by calculating the average rates weighted by the cohort number. With RT alone, the following rates were estimated: 87\% biochemical failure, 34\% local failure (LF), 48\% distant metastasis (DM), 38\% overall survival (OS), and 27\% disease-specific mortality (DSM). Those associated with ADT alone were 74\% BCF, 54\% OS, and 25\% DSM, which appeared to be better than those of RT alone. The addition of ADT to RT produced a notable local and systemic effect, regardless of ST- or LT-ADT. The LF rate decreased from 34\% with RT alone to 21\% with ST-ADT and further to $15 \%$ with LT-ADT. The DM and DSM rates also showed a similar trend among RT alone, RT+ST-ADT, and RT+LT-ADT. The combination of RT+LT-ADT resulted in the best long-term clinical outcomes, indicating that both RT and ADT are important parts of multimodal therapy.

Keywords: Locally advanced Prostate cancer, Multimodal therapy, Radiotherapy, Androgen deprivation therapy

\section{Introduction}

Prostate cancer is the most common malignancy in men in Western countries [1]. The incidence of prostate cancer in the Asia-Pacific region continues to increase [2]. In 2013, prostate cancer was the fifth most common cancer in men in South Korea, and the estimated numbers of new cases and deaths were approximately 9,500 and 1,600, respectively [3]. Since the introduction of prostate-specific antigen (PSA) testing, the proportion of locally advanced prostate cancer (LAPC) at diagnosis has decreased over the last two decades; however, patients presenting with LAPC are difficult to treat, as the optimal treatment method remains controversial.

LAPC is defined as histologically proven prostatic adenocarcinoma that spreads to the area outside of the prostate gland through the capsule (T3a), seminal vesicles (T3b), or adjacent organs (T4), such as the bladder or rectum. Radiotherapy (RT) had been the mainstay treatment for LAPC in the past. In recent years, there has been a significant improvement in outcome using the combination of RT and androgen deprivation therapy (ADT) $[4,5]$. To guide prostate cancer treatment, prognostic stage grouping (low-, intermediate-, and high-risk groups), which is based on Gleason score, the initial PSA level at the time of diagnosis, and anatomical stage, has long been used. Accordingly, the

Received 14 June 2017, Revised 25 July 2017, Accepted 11 August 2017.

Correspondence: Kwan Ho Cho, MD, The Proton Therapy Center, Research Institute and Hospital, National Cancer Center, 323 Ilsan-ro, Ilsandong-gu, Goyang 10408, Korea. Tel: +82-31-920-1720, Fax: +82-31-920-0149, E-mail: kwancho@ncc.re.kr

(c) This is an Open Access article distributed under the terms of the Creative Commons Attribution Non-Commercial License (http://creativecommons.org/ licenses/by-nc/4.0/) which permits unrestricted non-commercial use, distribution, and reproduction in any medium, provided the original work is properly cited.

www.e-roj.org 
recommended treatment and outcome report have been based on the risk group rather than the anatomical stage. Therefore, it is very difficult to determine the impact of disease extent based on anatomical stage. In this review, we define the individual roles of RT alone or its combination with short-term ADT (ST-ADT) or long-term ADT (LT-ADT), which have been used in non-surgical multimodal therapy for T3-4 LAPC.

\section{Literature Search Criteria}

A systematic review of studies retrieved from PubMed and MEDLINE published from February 2000 to February 2017 was performed. The search terms included 'locally advanced prostate cancer' or 'T3 prostate cancer' for reports published in English. The inclusion criteria were as follows: either randomized or non-randomized, the cohort with cT3-4 disease was greater than $50 \%$ of the study population, and long-term outcome results of 10 years or longer.

\section{Radiotherapy alone in the Management of LAPC}

The long-term clinical outcomes of RT alone for patients with LAPC were available in three randomized controlled trials, in which the original purpose of the trial was to compare RT alone with RT+ADT in an unfavorable group of patients [4-10] (Table 1). Radiation Therapy Oncology Group protocol 8531 (RTOG 8531) $[4,6,7]$ was designed to compare RT alone with RT+lifelong adjuvant androgen suppression (using goserelin) in an unfavorable group of patients with prostate cancer. Patients eligible for RTOG 8531 were those with a palpable primary tumor extending beyond the prostate (Clinical Stage T3) or those with regional Iymphatic involvement (26\%). Patients who had undergone prostatectomy were also eligible if penetration through the prostatic capsule to the margin of resection was observed and/or for those with seminal vesicle involvement, which accounted for 14\% of the cohort. Goserelin was permitted at relapse for those treated with RT alone. RTOG $8610[8,9]$ tested the hypothesis that STADT for 4 months before and during radiotherapy for LAPC may, by reducing the tumor bulk and enhancing the killing of tumor cells, improve locoregional control and ultimately survival compared with RT alone. Eligible patients were those with bulky tumors (T2-T4) with or without pelvic lymph node involvement and without evidence of distant metastasis. Bulky disease $\left(>25 \mathrm{~cm}^{2}\right)$ was excluded for RTOG 8531 [4], but was included for RTOG 8610 [8,9]. The European Organization for Research and Treatment of Cancer (EORTC) $22863[5,10]$ performed a randomized phase 3 trial assessing the benefits of LT-ADT for 36 months with a luteinizing hormone-releasing hormone (LHRH) agonist compared with RT in patients with a higher risk of metastases. Eligible patients were younger than 80 years and had newly diagnosed, histologically proven T1-2 prostatic adenocarcinoma with World Health Organization (WHO) histological grade 3 (10\%) or T3-4 prostatic adenocarcinoma of any histological grade (90\%), and WHO performance status of $0-2$. In these three trials, more than 900 patients received RT alone and more than $70 \%$ of these patients had T3-4 disease. The median ages were 70-71 years. The RT doses applied to the prostate target ranged from 65 to $70 \mathrm{~Gy}$. The presence of nodal disease varied from 3\% (EORTC 22863) to 26\% (RTOG 8531). RTOG 8531 defined biochemical failure (BCF) as a case of PSA $\geq 1.5 \mathrm{ng} / \mathrm{mL}$, which is slightly stricter than that defined by RTOG 8610 (PSA $\geq 2.0$ $\mathrm{ng} / \mathrm{mL}$ ). EORTC 22863 did not report the rate of BCF. The 10year long-term clinical outcomes after RT alone in these trials were reported as 80\%-91\% BCF, 24\%-38\% local failure (LF), 39\%-70\% distant metastasis (DM), 34\%-40\% overall survival (OS), and 22\%-36\% disease-specific mortality (DSM). BCF was more frequently observed in RTOG 8531 trials than in RTOG 8610 trials, which could be due to the different definitions of BCF and more patients with nodal disease being included in the RTOG 8531 study. The DM rates varied widely; however, the OS and DSM rates were relatively consistent among studies, ranging from $22 \%$ to $36 \%$.

Regarding radiation-induced toxicity, acute grade 3 toxicity was reported in 4\% of patients who received RT alone in RTOG 8531 [4], and late grade 3 toxicities were reported in $8 \%$ of patients $[8,9]$.

\section{ADT alone in the Management of LAPC}

The major benefit of ADT is likely related to the control of occult micrometastases, with some benefits related to delayed progression of local disease at the prostate [11]. Lifelong ADT consisting of either bilateral orchiectomy or LHRH agonists can continue until disease progression or death. The long-term results after ADT alone for patients with LAPC were available in two randomized controlled trials [12-14] (Table 2). In the National Cancer Institute of Canada Clinical Trial Group (NCIC CTG) and Medical Research Council (MRC) trial [12,13], 1,205 patients were randomly assigned (602 in the ADT alone group and 603 in the RT+LT-ADT group). Eligible patients were those with locally advanced (cT3 or T4) prostate cancer $(n=1,057)$ 
Table 1. Radiotherapy alone in the management of LAPC

\begin{tabular}{|c|c|c|c|c|}
\hline & RTOG $8531[4,6,7]$ & RTOG $8610[8,9]$ & EORTC $22863[5,10]$ & Total \\
\hline Type of study & III & III & III & \\
\hline \multicolumn{5}{|l|}{ Patients' characteristics } \\
\hline Number of patients & 488 & 232 & 208 & 928 \\
\hline Median age (yr) & - & 71 & 70 & 70-71 \\
\hline T3-4 (\%) & $>70$ & 70 & 89 & $70-89$ \\
\hline (+)N (\%) & 26 & 9 & 3 & $3-26$ \\
\hline$G S \geq 8$ & 32 & 30 & - & $30-32$ \\
\hline iPSA $\geq 20$ & - & - & 73 & - \\
\hline \multicolumn{5}{|l|}{ Treatment } \\
\hline RT (Gy) & $65-70$ & $65-70$ & 70 & $65-70$ \\
\hline Median follow-up (yr) & 7.6 & 13.2 & 9.1 & $7.6-13.2$ \\
\hline \multicolumn{5}{|c|}{ Clinical outcomes at 10 years (\%) } \\
\hline $\mathrm{BCF}$ & 91 & 80 & - & $80-91$ \\
\hline LF & 38 & - & 24 & $24-38$ \\
\hline DM & 39 & 47 & 70 & $39-70$ \\
\hline OS & 39 & 34 & 40 & $34-40$ \\
\hline DSM & 22 & 36 & 30 & $22-36$ \\
\hline
\end{tabular}

LAPC, locally advanced prostate cancer; RTOG, Radiation Therapy Oncology Group; EORTC, European Organization for Research and Treatment of Cancer; GS, Gleason score; iPSA, serum total prostate-specific antigen at presentation; RT, radiation treatment; BCF, biochemical failure; LF, local failure; DM, distant metastasis; OS, overall survival; DSM, disease specific mortality.

Table 2. ADT alone in the management of LAPC

\begin{tabular}{|c|c|c|c|}
\hline & NCIC CTG/MRC $[12,13]$ & SPCG-7/SFU0-3 [14] & Total \\
\hline Type of study & III & III & \\
\hline \multicolumn{4}{|l|}{ Patients' characteristics } \\
\hline Number of patients & 602 & 439 & 1,041 \\
\hline Median age (yr) & 70 & 66 & $66-70$ \\
\hline T3-4 (\%) & 87 & 79 & $79-87$ \\
\hline$(+) \mathrm{N}(\%)$ & - & - & - \\
\hline$G S \geq 8$ & 36 & - & - \\
\hline iPSA $\geq 20$ & 63 & 40 & $40-63$ \\
\hline \multicolumn{4}{|l|}{ Treatment } \\
\hline RT (Gy) & - & - & - \\
\hline ADT duration (mo) & Lifelong & Lifelong & Lifelong \\
\hline Median follow-up (yr) & 8 & 7.6 & $7.6-8$ \\
\hline \multicolumn{4}{|c|}{ Clinical outcomes at 10 years (\%) } \\
\hline $\mathrm{BCF}$ & 73 & 75 & $73-75$ \\
\hline LF & - & - & - \\
\hline DM & - & - & - \\
\hline OS & 49 & 60 & $49-60$ \\
\hline DSM & 25 & 24 & $24-25$ \\
\hline
\end{tabular}

ADT, androgen deprivation therapy; LAPC, locally advanced prostate cancer; NCIC CTG, National Cancer Institute of Canada Clinical Trials Group; MRC, Medical Research Council; SPCG-7, Scandinavian Prostate Cancer Group's Study VII; SFU0-3, Swedish Association for Urological Oncology 3; GS, Gleason score; iPSA, serum total prostate-specific antigen at presentation; RT, radiation treatment; BCF, biochemical failure; LF, local failure; DM, distant metastasis; OS, overall survival; DSM, disease specific mortality. 
or organ-confined disease (T2) with either a PSA concentration more than $40 \mathrm{ng} / \mathrm{mL}(n=119)$ or a PSA concentration more than $20 \mathrm{ng} / \mathrm{mL}$ and a Gleason score of 8 or higher $(n=25)$. In the Scandinavian Prostate Cancer Group Study No. 7/The Swedish Association for Urological Oncology-3 (SPCG-7/SFUO3) trial [14], 875 patients with LAPC (T3; 78\%; PSA <70; NO; $\mathrm{MO})$ were randomly assigned to endocrine treatment alone (3 months of total androgen blockade followed by continuous endocrine treatment using flutamide; 439 patients) or to the same endocrine treatment combined with RT (436 patients) [14]. The total number of patients randomized to receive ADT alone in these two trials was over 1,000. Bilateral orchiectomy was performed in $7 \%$ of patients in the NCIC CTG/MRC trial $[12,13]$. Node-positive disease was excluded after radiological or surgical evaluation. The median age ranged from 66 to 70 years, and T3-4 disease affected $79 \%$ to $87 \%$ of the population. Both studies used the BCF definition reported by the American Society for Therapeutic Radiology and Oncology (ASTRO) Phoenix (nadir $+2.0 \mathrm{ng} / \mathrm{mL}$ ). The 10 -year clinical outcomes of ADT alone in these trials were $73 \%-75 \% B C F_{1}$ 49\%-60\% OS, and 24\%-25\% DSM, with no reports on LF or DM. Without taking into consideration the differences in patient characteristics, the results were similar to those of RT alone (80\%-91\% BCF and 22\%-36\% DSM), with the exception of OS (49\%-60\% vs. $34 \%-40 \%)$. It is important to note, however, that the median age was 66 years for the ADT alone group compared with 70-71 years for the RT alone group.

In terms of treatment-related toxicity, sexual dysfunction (including impotence or loss of libido) was observed in up to $81 \%$ of patients with lifelong ADT use [14]. The five most frequent grade 3 or higher treatment-related toxicities were impotence/libido (29\%), hot flushes (8\%), urinary frequency $(4 \%)$, ischemia (3\%), and hypertension (3\%) in NCIC CTG/MRC $[12,13]$.

\section{RT with Short-Term ADT in the Management of LAPC}

The combination of RT and ADT has been tested by a number of researchers with the aim of enhancing the treatment effects on LAPC. Because both treatment methods have their own advantages and disadvantages (as a local therapy or systemic therapy), it is considered a reasonable approach to combine them for the treatment of LAPC. Arbitrarily, a 4-6-month prescription of ADT, usually given as a neoadjuvant, is considered short term. Three studies (two phase III trials and one retrospective series) are summarized in Table $3[8,9,15-$
17]. The purpose of RTOG 8610 was described in the previous section. Half of the study population $(n=224)$ was randomized to receive RT+ST-ADT composed of goserelin ( $3.6 \mathrm{mg}$ every 4 weeks) and flutamide (250 mg three times a day for 2 months) before and during RT $[8,9]$. RTOG 9202 was designed to compare ST-ADT (4 months) to LT-ADT (28 months) in addition to RT in patients with locally advanced-stage (T2c-4) prostate cancer and a PSA level of less than $150 \mathrm{ng} / \mathrm{mL}[15,16]$. After receiving ST-ADT and RT, a total of 1,554 patients were randomly assigned to receive 24-month ADT ( $n=758)$ vs. no more ADT ( $n=763$ ). Zelefsky et al. [17] retrospectively analyzed the clinical outcomes of RT+ST-ADT (3 months) in cT3 patients $(n=296)$. In their study, patients who exhibited seminal vesicle invasion (cT3b) accounted for $56 \%$ of all patients, and ST-ADT before and during RT was given in $64 \%$ of patients, and adjuvant ADT was not administered. A total RT dose of 65-70 Gy was applied to the prostate target (40-50 Gy to the whole pelvis and a 20-25 Gy boost to the prostate) in RTOG studies. However, in the study by Zelefsky et al. [17], a total of 66-86.4 Gy was delivered to the prostate target without whole pelvis treatment.

A total of 1,283 patients were treated with RT+ST-ADT, with the median age ranging from 68 to 72 years. BCF was defined as the PSA nadir + $2 \mathrm{ng} / \mathrm{mL}$. However, for RTOG $9202[15,16]$, BCF was defined using the ASTRO consensus definition (three consecutive increases in PSA level). The 10-year BCF, LF, DM, OS, and DSM rates were 63\%-68\%, 17\%-22\%, 23\%-36\%, 43\%-65\%, and 15\%-23\%, respectively. All clinical endpoints after RT+ST-ADT were consistently better than those of RT alone or ADT alone (except OS) throughout the studies. ST-ADT therapy appeared to enhance the local and systemic effects, as evidenced by the decreased rates of LF (from 24\%-38\% to $17 \%-22 \%$ ) and DM (from 39\%-70\% to $23 \%-36 \%$ ) by the addition of ST-ADT, with a subsequent reduction in DSM. The RTOG 8610 study $[8,9]$ revealed that the addition of 4 months of neoadjuvant ADT before RT was associated with a $26 \%$ improvement in the 10-year DSM and an increase of 1.4 years in the median survival time compared with those for RT alone.

With respect to radiation-induced toxicity, the rate of acute toxicity was reported as 2\%-9\% for grade 3 and approximately $1 \%$ for grade 4 . Late radiation-induced toxicity was observed in 4\%-8\% for grade 3 and 1\% for grade $4[8,9,15-17]$. After 10 years, fatal cardiac events occurred in $12.5 \%$ of the study cohort; however, the addition of ST-ADT to RT did not increase the risk of radiation-related toxicities or fatal cardiac events compared to RT alone $[8,9]$. 
Table 3. Radiotherapy with short-term ADT in the management of LAPC

\begin{tabular}{|c|c|c|c|c|}
\hline & $\begin{array}{c}\text { RTOG } 8610 \\
{[8,9]}\end{array}$ & $\begin{array}{c}\text { RTOG } 9202 \\
{[15,16]}\end{array}$ & $\begin{array}{c}\text { Zelefsky et al. } \\
\text { [17] }\end{array}$ & Total \\
\hline Type of study & III & III & $\mathrm{R}$ & \\
\hline \multicolumn{5}{|l|}{ Patients' characteristics } \\
\hline Number of patients & 224 & 763 & 296 & 1,283 \\
\hline Median age (yr) & 70 & 70 & 68 & $68-72$ \\
\hline T3-4 (\%) & 70 & 55 & 100 & $55-100$ \\
\hline$(+) \mathrm{N}(\%)$ & 7 & 4 & - & \\
\hline$G S \geq 8$ & 26 & 49 & 30 & $26-49$ \\
\hline iPSA $\geq 20$ & - & $>33$ & 40 & - \\
\hline \multicolumn{5}{|l|}{ Treatment } \\
\hline RT (Gy) & $65-70$ & $65-70$ & $66-86$ & $65-70$ \\
\hline ADT duration (mo) & 4 & 4 & 3 & $3-4$ \\
\hline Median follow-up (yr) & 11.9 & 11.3 & 8 & $8-11.9$ \\
\hline \multicolumn{5}{|c|}{ Clinical outcomes at 10 years (\%) } \\
\hline $\mathrm{BCF}$ & 65 & 68 & 63 & $63-68$ \\
\hline LF & - & 22 & 17 & $17-22$ \\
\hline DM & 35 & 23 & 36 & $23-36$ \\
\hline OS & 43 & 52 & 65 & $43-65$ \\
\hline DSM & 23 & 15 & 17 & $15-23$ \\
\hline
\end{tabular}

ADT, androgen deprivation therapy; LAPC, locally advanced prostate cancer; RTOG, Radiation Therapy Oncology Group; GS, Gleason score; iPSA, serum total prostate-specific antigen at presentation; RT, radiation treatment; $B C F$, biochemical failure; $L F$, local failure; $D M$, distant metastasis; OS, overall survival; DSM, disease specific mortality.

Table 4. Radiotherapy with long-term ADT in the management of LAPC

\begin{tabular}{|c|c|c|c|c|c|c|}
\hline & $\begin{array}{c}\text { RTOG } 8531 \\
{[4,6,7]}\end{array}$ & $\begin{array}{c}\text { RTOG } 9202 \\
{[15,16]}\end{array}$ & $\begin{array}{c}\text { EORTC } 22863 \\
\quad[5,10]\end{array}$ & $\begin{array}{c}\mathrm{NCIC} \mathrm{CTG/MRC} \\
{[12,13]}\end{array}$ & $\begin{array}{c}\text { SPCG-7/SFU0-3 } \\
{[14]}\end{array}$ & Total \\
\hline Type of study & III & III & III & III & III & \\
\hline \multicolumn{7}{|l|}{ Patients' characteristics } \\
\hline Number of patients & 489 & 758 & 207 & 603 & 436 & 2,493 \\
\hline Median age (yr) & - & 70 & 71 & 70 & 66 & $66-71$ \\
\hline T3-4 (\%) & $>70$ & 55 & 91 & 88 & 77 & $55-91$ \\
\hline$(+) \mathrm{N}(\%)$ & 29 & 3 & 4 & - & - & $4-29$ \\
\hline $\mathrm{GS} \geq 8$ & 32 & 46 & - & 36 & - & $32-46$ \\
\hline iPSA $\geq 20$ & - & $>33$ & 72 & 64 & 40 & $40-72$ \\
\hline \multicolumn{7}{|l|}{ Treatment } \\
\hline RT (Gy) & $65-70$ & $65-70$ & 70 & $65-69$ & 70 & 65-70 \\
\hline ADT duration (mo) & Lifelong & 28 & 36 & Lifelong & Lifelong & 28-lifelong \\
\hline Median follow-up (yr) & 7.6 & 11.3 & 9.1 & 8 & 7.6 & 7.6-9.1 \\
\hline \multicolumn{7}{|c|}{ Clinical outcomes at 10 years (\%) } \\
\hline $\mathrm{BCF}$ & 69 & 52 & - & 37 & 36 & $36-69$ \\
\hline LF & 23 & 12 & 6 & - & - & $6-23$ \\
\hline DM & 24 & 15 & 49 & - & - & $15-49$ \\
\hline OS & 49 & 54 & 58 & 55 & 70 & $49-70$ \\
\hline DSM & 16 & 11 & 10 & 15 & 12 & $10-16$ \\
\hline
\end{tabular}

ADT, androgen deprivation therapy; LAPC, locally advanced prostate cancer; RTOG, Radiation Therapy Oncology Group; NCIC CTG, National Cancer Institute of Canada Clinical Trials Group; MRC, Medical Research Council; SPCG-7, Scandinavian Prostate Cancer Group's Study VII; SFUO-3, Swedish Association for Urological Oncology 3; GS, Gleason score; iPSA, serum total prostate-specific antigen at presentation; RT, radiation treatment; BCF, biochemical failure; LF, local failure; DM, distant metastasis; OS, overall survival; DSM, disease specific mortality. 


\section{RT with Long-Term ADT in the Management of LAPC}

When LAPC patients are treated with RT+ADT, LT-ADT is generally defined as the administration of ADT over 2 years, usually prescribed as an adjuvant. A total of five phase III trials have reported long-term follow-up results from approximately 2,500 patients [4-7,10,12-16] (Table 4). As described previously, the purposes of those five studies were to compare the clinical outcomes of LT-ADT+RT with RT alone (RTOG 8531 and EORTC 22863) [4-7,10], or with ADT alone (NCIC CTG/MRC and SPCG7/SFU0-3) [12-14] or with ST-ADT (RTOG 9202) $[15,16]$ in LAPC patients. In most studies, the median age was around 70 years but, in SPCG-7/SFU0-3, it was 66 years [14]. RTOG 8531 enrolled a larger proportion of node-positive disease (29\%) cases than the others $(3 \%-4 \%)[4,6,7]$. Patients with locally advanced stage (cT3-4) accounted for over 70\% of the cohort, except for RTOG 9202 (cT2c 45\%, cT3-4 55\%). A total dose of 65-70 Gy was delivered to the prostate target (approximately 45-50 Gy of pelvic RT followed by 20-25 Gy of prostate boost), but pelvic RT was not mandatory in SPCG-7/SFU0-3. The duration of LT-ADT varies from 28 months to lifelong. As shown in Fig.1, RT+LT-ADT produced more favorable clinical outcomes than RT alone, ADT alone, and RT+ST-ADT. In the three studies $[4-7,10,15,16]$ that defined the role of LT-ADT in multimodality therapy, there were remarkable improvements in clinical outcomes, as evidenced by the decrease in 10year rates of BCF (from 80\%-91\% to 52\%-69\%), LF (from $24 \%-38 \%$ to $6 \%-23 \%$ ), DM (from $39 \%-70 \%$ to $15 \%-49 \%$ ), and DSM (from 22\%-36\% to 10\%-16\%), and an increase in the 10-year rate of OS (from 34\%-40\% to 49\%-58\%) (all from without to with LT-ADT, respectively). Likewise, in two randomized trials [12-14] that defined the role of RT in multimodality therapy, there were remarkable improvements in clinical outcomes, as evidenced by the decreased rates of BCF (from 73\%-75\% to 26\%-37\%) and DSM (from 24\%-25\% to 12\%-15\%), and an increase in the rate of OS (from 49\%-60\% to 55\%-70\%) (all from without to with RT, respectively). In NCIC CTG/MRC $[12,13]$, the hazard ratio for BCF by the addition of RT to ADT was 0.31 (95\% confidence interval, 0.27-0.37) compared with ADT alone. The OS improved from 66\% without RT to $74 \%$ with it ( $p=0.03$ ) after 7 years. The SPCG-7/SFU0-3 study demonstrated the highest 10 -year OS (70\%) in relatively young patients (median age, 66 years) [14]. Compared with ADT alone, the addition of RT halved the rate of DSM in NCIC CTG/MRC and SPCG-7/SFU0-3 after 10 years. These two studies prove that RT is a crucial part of multimodality therapy in the management of LAPC. Together, the OS improved from 49\%-60\% without RT to 55\%-70\% with it after 10 years. In summary, compared to RT alone or ADT alone, LT-ADT+RT was associated not only with reductions in LF and DM rates, but also with a statistically significant improvement in OS [4-7, 10,12-16]. In addition, in RTOG 9202 [15,16], which compared ST-ADT with LT-ADT+RT, LT-ADT caused a significant improvement in all endpoints, except for OS, which was limited to a subgroup of patients with a Gleason score between 8 and 10.

Treatment-related toxicity after RT combined with LTADT could be caused by RT and ADT either additively or synergistically. With respect to radiation-induced toxicity, 7\%

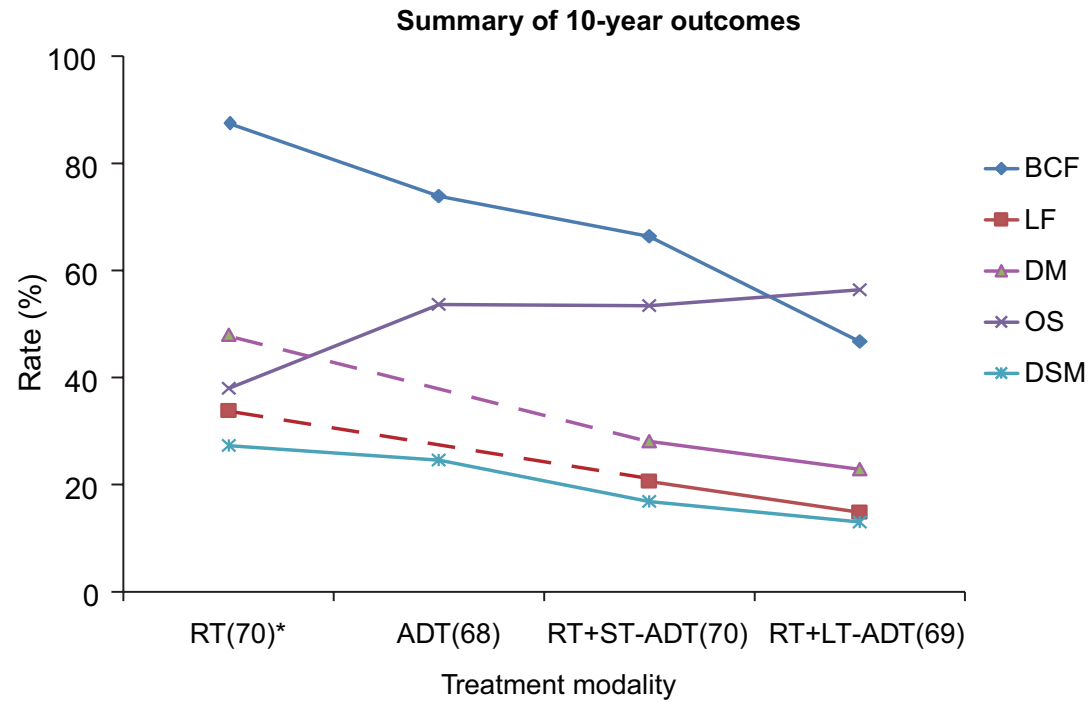

Fig. 1. The role of individual treatment and their combination in multimodal therapy for locally advanced prostate cancer. The y-axis represents weighted average rates by the cohort number. $R T$, radiotherapy; ADT, androgen deprivation therapy; ST-ADT, short-term ADT LT-ADT, long-term ADT; BCF, biochemical failure; LF, local failure; DM, distant metastasis: OS, overall survival; DSM, disease specific mortality. ${ }^{*}$ The number in parentheses is median age. 
of grade 3 and 1\% of grade 4 acute radiation-induced toxicity was reported $[15,16]$. Gastrointestinal toxicity was more frequently observed in subjects who received RT+LT-ADT than ADT alone (most were grades 1-2) [12,13], and the difference in moderate or severe GI symptoms was not significant (1\%-3\%) [14]. Compared with ST-ADT+RT, LT-ADT increased radiation-induced late toxicity, with 7\% of grade 3 and $3 \%$ of grade 4 late radiation-induced toxicity [18]. Regarding the toxicity related to LT-ADT, the five most frequent grade 3 or higher toxicities were impotence/libido (33\%), hot flushes (5\%), urinary frequency (7\%), ischemia (5\%), and hypertension (4\%) $[12,13]$. In RTOG 9202, one patient died as a result of chemical hepatitis during ADT $[15,16]$. LT-ADT+RT did not significantly increase the risk of fatal cardiac events compared to RT alone, ADT alone, or ST-ADT+RT $[5,13,16]$.

\section{Relative Contributions of Individual Treatments}

Owing to disparities in pre-treatment patient characteristics, the study endpoints and definitions, and the salvage treatment modality for recurrence after initial treatment, it is difficult to compare clinical outcomes among studies. Despite these limitations, a trend of 10 -year clinical outcomes was roughly estimated from published papers by calculating the average rates weighted by the number of subjects to determine the roles of individual treatments used in multimodal therapy for LAPC (Fig. 1). With RT alone, the following rates were estimated: $87 \%$ BCF, 34\% LF, 48\% DM, 38\% OS, and 27\% DSM. Those associated with ADT alone were 74\% BCF, 54\% OS, and 25\% DSM, which appeared to be better than those of RT alone. The OS was higher in patients who were treated with ADT alone compared with RT alone (54\% vs. 38\% at 10 years, respectively), although it is important to note that the median age was younger in those of ADT alone than those of RT alone (68 vs. 70 years). Regardless, the DSM was similar between two groups. The addition of ADT to RT has shown notable local and systemic effects, regardless of ST- or LT-ADT. With RT+ST-ADT, the following rates were observed: 66\% BCF, 21\% LF, 28\% DM, $53 \%$ OS, and 17\% DSM; the corresponding rates of RT+LTADT were 47\%, 15\%, 23\%, 56\%, and 13\%, respectively. As evidenced by the results concerning either ST-ADT or LT-ADT, the DM rate was profoundly reduced from 48\% with RT alone to $28 \%$ with ST-ADT and to $23 \%$ with LT-ADT. Interestingly, the LF rate was also decreased from 34\% with RT alone to 21\% with ST-ADT and further to 15\% with LT-ADT. Therefore, we conclude that systemic therapy (ADT) can reduce the local failure rate in the long term. In the same manner, one can postulate that local therapy (RT) can reduce the rate of DM in the long term by applying radiation to the primary source of metastases in the prostate. However, we were not able to prove this postulation because no DM data were reported in the studies of ADT alone, which is required for the comparison of ADT with or without RT. Although the 10-year OS rate after treatment varied widely (34\%-70\%) and younger age at diagnosis was the strongest factor for long-term OS, RT+LTADT showed a favorable 10-year OS (over 50\%), even though the median age at diagnosis in most studies was 70 years old, which was consistently better than in the control arms of RT alone or ADT alone or RT+ST-ADT in randomized trials. With respect to treatment related toxicity, acute and late toxicities are usually mild to moderate with rare severe ones even in the combined modality.

\section{Other Issues}

With advances of technology in radiation therapy, dose escalation becomes possible to the target without increasing toxicity by using intensity modulated radiotherapy or image guided radiotherapy. There are several randomized clinical trials demonstrating clinical benefits in patients with prostate cancer [19-21]. However, patients eligible for these trials were those with early stage of cancer but not those with LAPC (usually $<20 \%$ of the cohort had LAPC). The escalated doses (78-80 Gy) were associated with an improvement in the biochemical control, but not in the OS [19-21], compared with those for conventional doses (70 Gy). The role of hypofractionated RT was also studied in many trials $[22,23]$. Eligible patients for these trials were those with early stage (low- or intermediated-risk group) rather than those with LAPC $[22,23]$. The role of brachytherapy boost in addition to external beam RT (EBRT) for patients with intermediate- and high-risk has been investigated in the ASCENDE-RT phase III trial [2426]. A total of 398 patients (approximately 30\% of the cohort were those with LAPC) were randomized to a standard arm with 12 months of ADT, pelvic irradiation to $46 \mathrm{~Gy}$, followed by a dose-escalated EBRT boost to $78 \mathrm{~Gy}$, or an experimental arm that substituted a low-dose-rate prostate brachytherapy boost (LDR-PB). Compared with 78 Gy EBRT, patients randomized to the LDR-PB were twice as likely to be free of BCF at a median follow-up of 6.5 years. However, there was no difference in OS between two arms, but the LDR-PB caused significantly more deteriorated urinary function in both toxicity rate and quality of life scale [24-26]. In summary, there are no trials to 
define the role of dose-escalation in RT using conventional fractionation, hypo-fractionation, or brachytherapy-boost solely for patients with LAPC in the present time; hence, their roles have not been well defined yet. It is important to note that the rate of 23\% DM were higher than those of 15\% LF after the current standard care of RT+LT-ADT in patients with LAPC. Therefore, the escalation of systemic therapy is potentially more effective than the escalation of local therapy to improve the clinical outcome. There are couple of studies demonstrating an improvement of OS in their interim analyses by addition of docetaxel to ADT in high risk patients $[27,28]$. Likewise, the addition of abiraterone to ADT significantly increased overall survival in men with newly diagnosed, metastatic, castration-sensitive prostate cancer [29]. It is interesting to see whether those intensification of systemic therapy has a role for LAPC.

\section{Conclusions}

We reviewed the studies related to the roles of radiotherapy, androgen deprivation therapy, and their combination for LAPC. In conclusion, RT+LT-ADT results in the best long-term clinical outcomes, in which both RT and ADT are crucial parts of multimodal therapy. Further refinement of combined modality therapy needs to be explored.

\section{Conflict of Interest}

No potential conflict of interest relevant to this article was reported.

\section{Acknowledgments}

This work was supported by a National Cancer Center (Grant No. NCC-1610440).

\section{References}

1. Attard G, Parker C, Eeles RA, et al. Prostate cancer. Lancet 2016;387:70-82.

2. Baade PD, Youlden DR, Cramb SM, Dunn J, Gardiner RA. Epidemiology of prostate cancer in the Asia-Pacific region. Prostate Int 2013;1:47-58.

3. Oh CM, Won YJ, Jung KW, et al. Cancer Statistics in Korea: Incidence, Mortality, Survival, and Prevalence in 2013. Cancer Res Treat 2016;48:436-50.

4. Pilepich MV, Winter K, Lawton CA, et al. Androgen suppression adjuvant to definitive radiotherapy in prostate carcinoma: long-term results of phase III RTOG 85-31. Int J Radiat Oncol Biol Phys 2005;61:1285-90.

5. Bolla $M$, Van Tienhoven $G$, Warde $P$, et al. External irradiation with or without long-term androgen suppression for prostate cancer with high metastatic risk: 10-year results of an EORTC randomised study. Lancet Oncol 2010;11:1066-73.

6. Lawton CA, Winter K, Murray K, et al. Updated results of the phase III Radiation Therapy Oncology Group (RTOG) trial 8531 evaluating the potential benefit of androgen suppression following standard radiation therapy for unfavorable prognosis carcinoma of the prostate. Int J Radiat Oncol Biol Phys 2001;49:937-46.

7. Pilepich MV, Caplan R, Byhardt RW, et al. Phase III trial of androgen suppression using goserelin in unfavorableprognosis carcinoma of the prostate treated with definitive radiotherapy: report of Radiation Therapy Oncology Group Protocol 85-31. J Clin Oncol 1997;15:1013-21.

8. Pilepich MV, Winter $K_{1}$ John MJ, et al. Phase III radiation therapy oncology group (RTOG) trial 86-10 of androgen deprivation adjuvant to definitive radiotherapy in locally advanced carcinoma of the prostate. Int J Radiat Oncol Biol Phys 2001;50:1243-52.

9. Roach M 3rd, Bae K, Speight J, et al. Short-term neoadjuvant androgen deprivation therapy and external-beam radiotherapy for locally advanced prostate cancer: long-term results of RTOG 8610. J Clin Oncol 2008;26:585-91.

10. Bolla $M$, Collette $L$, Blank $L$, et al. Long-term results with immediate androgen suppression and external irradiation in patients with locally advanced prostate cancer (an EORTC study): a phase III randomised trial. Lancet 2002;360:103-6.

11. McLaughlin PW, Liss AL, Nguyen PL, et al. ACR Appropriateness Criteria locally advanced, high-risk prostate cancer. Am J Clin Oncol 2017;40:1-10

12. Warde $P$, Mason $M$, Ding $K$, et al. Combined androgen deprivation therapy and radiation therapy for locally advanced prostate cancer: a randomised, phase 3 trial. Lancet 2011;378:2104-11.

13. Mason MD, Parulekar WR, Sydes MR, et al. Final report of the intergroup randomized study of combined androgendeprivation therapy plus radiotherapy versus androgendeprivation therapy alone in locally advanced prostate cancer. J Clin Oncol 2015;33:2143-50.

14. Widmark $A_{1}$ Klepp O, Solberg A, et al. Endocrine treatment, with or without radiotherapy, in locally advanced prostate cancer (SPCG-7/SFU0-3): an open randomised phase III trial. Lancet 2009;373:301-8. 
15. Hanks GE, Pajak TF, Porter A, et al. Phase III trial of long-term adjuvant androgen deprivation after neoadjuvant hormonal cytoreduction and radiotherapy in locally advanced carcinoma of the prostate: the Radiation Therapy Oncology Group Protocol 92-02. J Clin Oncol 2003;21:3972-8.

16. Horwitz EM, Bae K, Hanks GE, et al. Ten-year follow-up of radiation therapy oncology group protocol 92-02: a phase III trial of the duration of elective androgen deprivation in locally advanced prostate cancer. J Clin Oncol 2008;26:2497-504.

17. Zelefsky MJ, Yamada Y, Kollmeier MA, Shippy AM, Nedelka MA. Long-term outcome following three-dimensional conformal/ intensity-modulated external-beam radiotherapy for clinical stage T3 prostate cancer. Eur Urol 2008;53:1172-9.

18. Brundage $M$, Sydes $M R$, Parulekar WR, et al. Impact of radiotherapy when added to androgen-deprivation therapy for locally advanced prostate cancer: long-term quality-oflife outcomes from the NCIC CTG PR3/MRC PR07 randomized trial. J Clin Oncol 2015;33:2151-7.

19. Kuban DA, Tucker $S L$, Dong $L$, et al. Long-term results of the M. D. Anderson randomized dose-escalation trial for prostate cancer. Int J Radiat Oncol Biol Phys 2008;70:67-74.

20. Beckendorf V, Guerif S, Le Prise E, et al. 70 Gy versus 80 Gy in localized prostate cancer: 5-year results of GETUG 06 randomized trial. Int J Radiat Oncol Biol Phys 2011;80:105663.

21. Zietman $A L$, DeSilvio $M L$, Slater JD, et al. Comparison of conventional-dose vs high-dose conformal radiation therapy in clinically localized adenocarcinoma of the prostate: a randomized controlled trial. JAMA 2005;294:1233-9.

22. Dearnaley D, Syndikus I, Mossop $H$, et al. Conventional versus hypofractionated high-dose intensity-modulated radiotherapy for prostate cancer: 5-year outcomes of the randomised, noninferiority, phase 3 CHHiP trial. Lancet Oncol 2016;17:1047-60.
23. Lee WR, Dignam JJ, Amin MB, et al. Randomized phase iii noninferiority study comparing two radiotherapy fractionation schedules in patients with low-risk prostate cancer. J Clin Oncol 2016;34:2325-32.

24. Morris WJ, Tyldesley S, Rodda S, et al. Androgen Suppression Combined with Elective Nodal and Dose Escalated Radiation Therapy (the ASCENDE-RT Trial): an analysis of survival endpoints for a randomized trial comparing a low-dose-rate brachytherapy boost to a dose-escalated external beam boost for high- and intermediate-risk prostate cancer. Int J Radiat Oncol Biol Phys 2017;98:275-85.

25. Rodda S, Tyldesley S, Morris WJ, et al. ASCENDE-RT: an analysis of treatment-related morbidity for a randomized trial comparing a low-dose-rate brachytherapy boost with a doseescalated external beam boost for high- and intermediate-risk prostate cancer. Int J Radiat Oncol Biol Phys 2017;98:286-95.

26. Rodda S, Morris WJ, Hamm J, Duncan G. ASCENDE-RT: an analysis of health-related quality of life for a randomized trial comparing low-dose-rate brachytherapy boost with doseescalated external beam boost for high- and intermediate-risk prostate cancer. Int J Radiat Oncol Biol Phys 2017;98:581-9.

27. James N, Mason M. Docetaxel and/or zoledronic acid for hormone-naïve prostate cancer: first survival results from STAMPEDE [abstract]. J Clin Oncol 2015;33(Suppl):5001.

28. Sandler HM, Hu C, Rosenthal SA, et al. A phase III protocol of androgen suppression (AS) and 3DCRT/IMRT versus AS and 3DCRT/IMRT followed by chemotherapy (CT) with docetaxel and prednisone for localized, high-risk prostate cancer (RTOG 0521) [abstract]. J Clin Oncol 2015;33(Suppl):LBA5002.

29. Fizazi $K$, Tran N, Fein $L$, et al. Abiraterone plus prednisone in metastatic, castration-sensitive prostate cancer. N Engl J Med 2017;377:352-60. 